\title{
Assisting the Driver with Distance Estimation: Usability Evaluation of Graphical Presentation Alternatives for Local Traffic Events
}

\author{
Angela Mahr, Sandro Castronovo, Rafael Math, and Christian Müller \\ German Research Center for Artificial Intelligence, \\ Intelligent User Interfaces Department, Campus D 3_2, \\ 66123 Saarbrücken, Germany \\ \{angela.mahr, sandro.castronovo, rafael.math, \\ christian.mueller\}@dfki.de
}

\begin{abstract}
When integrating numerous in-car information and assistance systems, a consistent way of spatial distance presentation for drivers is required. A common practice is to use discrete textual information (e.g. 500 meters) in combination with a graphical bar representing relative spatial information. Hitherto there exists no design consistency with respect to bars for distance illustration. Contemporary solutions differ in terms of movement direction (upward vs. downward), composition type (decreasing vs. increasing), or alignment (horizontal vs. vertical). We conducted a driving simulator experiment to investigate user preferences, perceived location, and eye gaze data for a meaningful subset of bars in a dynamic scenario. When approaching a traffic event (road works), one out of four vertical bar alternatives indicated the current distance. Subsequently, the associated horizontal bar type (decreasing or increasing) visualized the driver's spatial progress within the road works section. Our results indicate, that drivers prefer upwards-moving approach bars and progress bars decreasing from left to right. Eye-tracking data supports usage of decreasing bars instead of increasing bars. Accordingly, we elaborated an initial version of design guidelines for bars representing relative spatial information for local events. On this basis we implemented approach and progress indicators, which were adopted for numerous use-cases in a large field operational test for Vehicle-2-X Communication.
\end{abstract}

Keywords: Distance, Assistance, Design, Usability Evaluation, In-car, Driving.

\section{Introduction}

During the last decades, awareness regarding intuitive driver interfaces has constantly increased. With a rising number of in-vehicle information systems (IVIS) and advanced driver assistant systems (ADAS) being integrated, coherent presentation of information is crucial for drivers to understand contents immediately. Although multimodal presentations open new options, common graphical displays will not become dispensable for automotive applications. Due to visual driver distraction while 
glancing at the display, it is necessary to reduce glancing times to a minimum by intuitive presentations. This is one fundamental design principle for interaction with IVIS [1].

One of the frequently required functionalities of in-car displays is spatial distance presentation, e.g. in today's navigation systems when a maneuver point comes up (e.g. turn right in $300 \mathrm{~m}$ ). In cases of events that can be pinpointed to a location, we use the term spatial approach visualization. Additionally, modern ADAS require visual presentation of spatial validity, e.g. visualizing the remaining section where a traffic sign is valid. In this case of traffic events having an areal extend, we name it spatial progress visualization. Although in general HCI progress is well researched [2-7], the automotive domain lacks both a common concept, as well as design guidelines for implementations of spatial approach and progress. We found a general tendency of numerical distance presentation (e.g. 300 meters) in combination with a graphical progress bar representing relative spatial information when investigating hitherto existing distance presentation in diverse system types. For presentation of spatial information, there exist many plausible variants, and unfortunately no design consensus could be observed. Nevertheless, three major design factors could be derived: First, there exist two general orientations of the bar (horizontal vs. vertical), second, the bar can be filled or emptied with some kind of filling (increase vs. decrease), and third, the movement direction can be manipulated (up vs. down or left vs. right respectively). Moreover, color-coding is sometimes used for urgency emphasis.

In this paper we examine these different design factors with the help of a user study. Our goal was to find parameter combinations, which are preferred by drivers, perceived fastest, and minimize glancing times. Accordingly, a suitable driving task was designed and implemented via the openDS driving simulator [8] and an eye tracker was used to measure glance durations while driving. Based on our results, we suggest general design guidelines for spatial approach and progress in the automotive domain. These guidelines have already been applied to a Human-Machine Interface used in a large field operational test with over 20 novel ADAS $\left(\operatorname{sim}^{\mathrm{TD}}\right)$.

\section{The $\operatorname{sim}^{\mathrm{TD}}$ HMI}

The $\operatorname{sim}^{\mathrm{TD}}$ project ${ }^{1}$ comprises one of the largest field operational tests for Vehicle-2-X Communication Networks (V2X) and its applications. In this domain, cars communicate with other entities using different wireless communication technologies where ' $\mathrm{X}$ ' denotes the communication partner. These are typically other cars, road infrastructure like signal lights, or traffic management authorities. $\operatorname{sim}^{\mathrm{TD}}$ investigates established V2X use-cases in real traffic scenarios around the Hessian city of Frankfurt/ Main, Germany with a fleet of 120 cars. One of the goals of the project is to evaluate the effect of V2X-based applications on traffic and driver. It is currently the largest field operational test in this domain. Drivers are not only advised "experts" to the $\operatorname{sim}^{\mathrm{TD}}$ system but also novices. Especially for this type of drivers it is of utmost

\footnotetext{
${ }^{1}$ http: / / www.simtd.de/
} 
importance that the Human-Machine Interface (HMI) of the system is intuitive, nondistractive and features a consistent integration of all use-cases. It consists of a main area where the most relevant information is displayed and an additional area in the upper part containing six slots for additional information of parallel applications. Using two virtual buttons, the driver can also access a map and an option screen (see Figure 1). The Automotive group at "double-blinded" is responsible for developing and evaluating the HMI concept in $\operatorname{sim}^{\mathrm{TD}}$ prior to the field operational test.

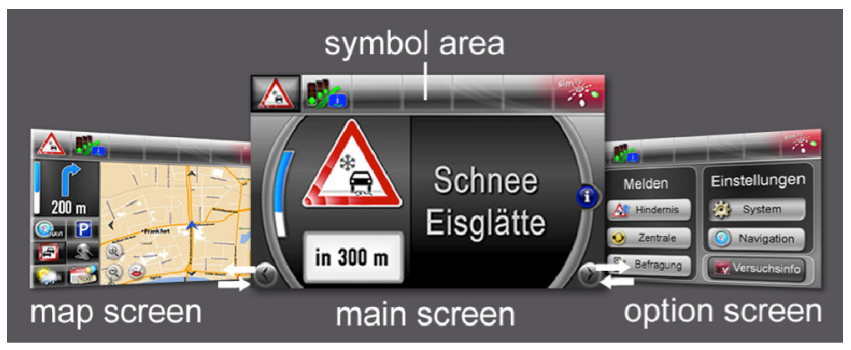

Fig. 1. The $\operatorname{sim}^{\mathrm{TD}}$ HMI depicting three virtual screens and symbol area

The V2X use-cases evaluated can be classified into three categories: Safety, traffic efficiency, and value added services. Many events belonging to these use-cases are location-based, i.e. they pinpoint to a certain geographical position. Moreover, some events are valid for a certain road segment, e.g. road works. Table 1 lists a shortened selection of use-cases, which are investigated in $\operatorname{sim}^{\mathrm{TD}}$. Altogether, there are over 25 use-cases tested within the project. We refer the interested reader to the full list of selected functions in [9]. Especially warning functions, e.g. congestion warning, need to be displayed way before the actual event. Those approaches to upcoming events should be presented to drivers in a consistent way for all use-cases. Besides text-only information, the use of graphical information via progress bars can be found in several navigation systems. We analyze different realizations and have a look what can be learned from general HCI research in the following section.

Table 1. Some selected $\operatorname{sim}^{\mathrm{TD}}$ use-cases involving approach and/or progress information

\begin{tabular}{|c|l|}
\hline Category & Use-case \\
\hline Safety & Lost Cargo Warning \\
\hline Safety & Congestion Ahead Warning \\
\hline Safety & Animals on the Road Warning \\
\hline Safety & Approaching Emergency Vehicle Warning \\
\hline Safety & Road Weather Warning \\
\hline Efficiency & Traffic Sign Assistant \\
\hline Efficiency & Road Works Information System \\
\hline
\end{tabular}

\section{Background}

Since the beginning of HCI research, design and display of progress is considered important and useful. For example, Myers in [7] concludes that especially for 
long-lasting tasks users prefer this kind of progress display. In traditional HCI, the design of progress was established even before graphical user interfaces were common. It was implemented using ASCII characters (cf. Figure 2a).

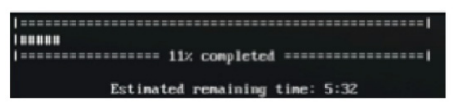

(a) ASCII

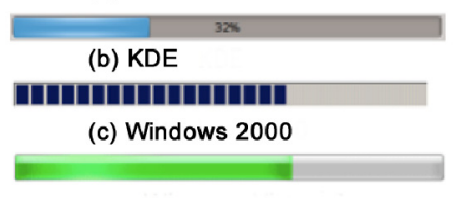

(d) Windows Vista / 7

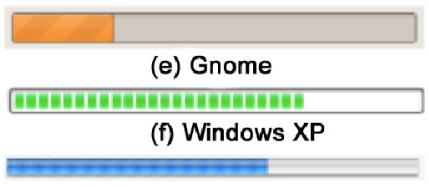

(g) Mac OS

Fig. 2. Design of progress in different Operating Systems (b)-(g) and in ASCII code (a)

Nowadays, horizontal filling bars (from left to right) constitute a common implementation on Desktop Operating Systems and mobile devices. With Windows 2000 and Windows XP, segmented bars were invented but replaced by continuous bars in Windows Vista/7 (Figure 2, (c), (f), (d)). Modern versions of all major Desktops come with an animation of the bars' fill color either "pulsating" from left to right (Windows) or "ribbed" from right to left, e.g. in Mac, see Figure 2, (g). One might think this change was invented because of advances in technology such as increased computing power or in order to make the graphical user interface more appealing. But interestingly, according to [3], this type of bars result in statistically significant shorter perceived duration times when compared to their static counterparts. Another approach is hiding the actual progress from the user and displaying another one, as investigated in [4]. Harrison et al. applied non-linear functions for progress bars and found that users have a strong preference for speed-ups towards the end. On the other side, according to their results, users tend to tolerate slow-downs at the beginning better than towards the end. For tasks with dynamic completion, like defragmenting a hard-drive, such a non-linear progress bar is considered useful. Such a progress bar, on the other hand, is not appropriate for linear tasks. Other approaches try to exploit colors or different kinds of modalities. For example in [2], six combinations of colors are considered: blue/red for progress bar foreground color and cyan/orange/gray for background color. Furthermore, [6] encode progress into a series of vibrotactile pulses while [5] use auditory cues. To conclude, so far, researchers above all tried to reduce the subjective perception of waiting time, either by visual means, or by applying non-linear functions to progress bars.

However, these strategies should only be applied, when progress of the actual task is hidden from the user. In the automotive domain this decoupling between actual and displayed spatial information is neither given nor desired. Quite contrary, it is essential that both coincide as exactly as possible, e.g. during a spatial approach to a maneuver point. Besides that, transferred to our domain, Harrison et al. suggest in [4] to represent linear tasks by linear progress. Also, the use of animations in the automotive context might induce visual distraction. 
Unlike in general HCI research, consistency with respect to design of spatial approach could not be observed between several manufacturers of navigation systems. Figure 3 depicts variants from different vendors. They vary in direction (decreasing vs. increasing) and movement (upwards vs. downwards). Even a single manufacturer employs increasing and decreasing bars in different models, see (f) and (g) in Figure 3. Falk (d) realizes segmented bars as arrows to indicate the filling direction. Several vendors provide no visual directional cue at all (NavigonMobile (f), TomTom (e)). This listing is not meant to be complete, but is supposed to reveal existing inconsistency found in different navigation systems as opposed to desktop progress bar design.

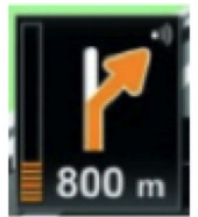

(a) Navigon $(\uparrow,>)$

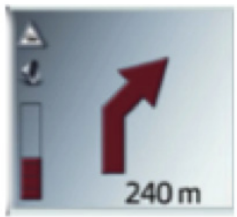

(b) Becker (,$>)$

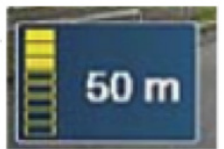

(c) Blaupunkt $(\uparrow,<)$

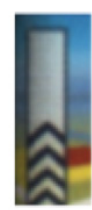

(d) Falk (个,>)

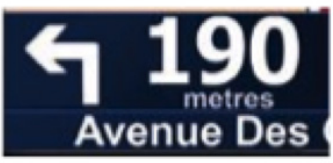

(e) TomTom (囚,囚)

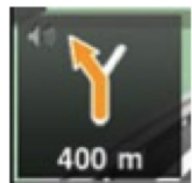

(f) NavigonMobile (囚,囚)

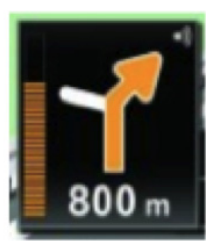

(g) Navigon $(\downarrow,<)$

Fig. 3. Design of spatial approach in different navigation systems. Legend: ' $\uparrow$ '=Upwards; ' $\downarrow$ '=Downwards; '>’=Increasing; ' $<$ '=Decreasing, ' $囚$ '=Feature not present.

Despite these differences, we could however observe several common criteria: All observed designs of spatial approach are realized as straight, vertical, and segmented bars. Minimum and maximum bar states could be inferred by a frame or similar means. Additional textual information (e.g. 500 meters) was presented in every design. We decided to reflect these shared criteria by setting them as preconditions in the design of experimental conditions. Accordingly, we used textual information in combination with a graphical bar representing relative spatial information in each condition. However, the observed inconsistencies in existing systems and a lack of literature on the issue in the automotive context lead to the conclusion that still many questions are unanswered when it comes to design of spatial approach and progress:

1. Do users find graphical approach and progress bars useful?

2. Which progress bar type is preferred (increasing vs. decreasing, upwards vs. downwards, use of colors, relevance of frame)?

3. Do different progress bar variants induce different visual distraction from the driving task (accessed by eye-tracking data or driving performance)?

The aim of the experiment presented here, was to answer the questions above and derive a universal guideline for designing consistent spatial approach and progress for 
in-vehicle information systems. It is important to notice that dynamic bars cannot be evaluated on static screenshots as it is for example impossible to infer movement direction or rate of changes. Furthermore, watching simply animated bars is not comparable with a driver (visually) engaged in a driving task using bar animations as relative spatial information for local events. On these grounds, we decided to conduct a driving simulator experiment with a mock spatial approach and progress scenario.

\section{Experiment}

\subsection{Participants}

24 participants participated in this experiment, 12 men and 12 women. Their age varied between 19 years and 59 years $($ mean $=37.0, \mathrm{SD}=15.1)$. All of them possessed a valid driver's license for at least two years. Half of the participants owned a navigation system and indicated to use it frequently, 2 participants indicated to use a navigation system occasionally, and 10 participants have only rarely used a navigation system before. Accordingly, no absolute novices took part. Participants were paid 10 Euros for approximately 1 hour of time.

\subsection{Apparatus}

The experiment setup consisted of a Logitech Momo steering wheel attached to a desk and two pedals on the floor, resembling the ergonomic condition of a driver in a car (cf. Figure 4). During the experiment, the participant's task was to steer and brake with these devices according to driving requirements. A computer flat screen (size 20 inch, resolution $1440 \times 1050$ pixel) presenting the driving simulation was set up on a desk with a distance of about 80 centimeters to the participant's head.

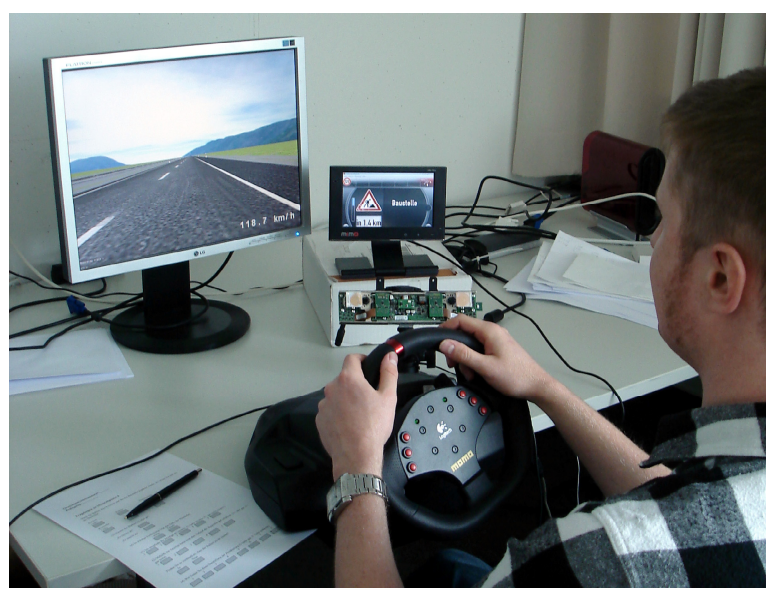

Fig. 4. Experimental Setup

White digits on the lower right side of the screen displayed the current vehicle speed. Their color turned into red, whenever drivers diverged from the currently prescribed maximum speed for more than 10 kilometers per hour. We used an open 
source driving simulation solution, which has recently been presented in [8]. For this experiment, several highway tracks were generated with each track including a road works section towards its end (see also Driving Task and Tracks section). In-vehicle information about an upcoming or current traffic situation was presented at the right hand side of the driver on a seven-inch MiMo screen. A Tobii eye tracker was installed below the small information screen for recording respective gazes onto this screen. A detailed description of the information screen and the presented bar variant combinations will be provided in the following section.

\subsection{Information Screen and Presented Bar Types}

Figure 5 depicts an example of all items displayed on the information screen.
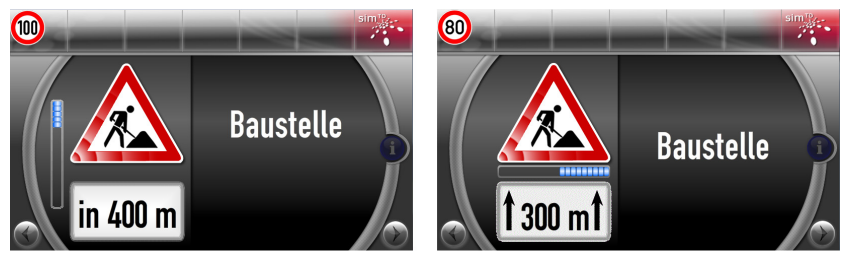

Fig. 5. Two screenshots of displays for the road works use-case. Distance is indicated in numerical form (in $400 \mathrm{~m} / 300 \mathrm{~m}$ ) accompanied by a graphical bar representing relative spatial information. The left side depicts a screenshot for approaching road works and the right side shows a presentation while driving within a road works section.

Besides the current speed limit in the upper left corner, the type of the upcoming traffic event (road works) is presented. Most important, numerical distance indicators combined with spatial bars were presented in all cases along the general lines of [10]. For approaching road works, a vertical bar was used and while driving through a section of road works, a horizontal bar indicated the current progress. Hence, the alignment of a bar itself would provide information whether a driver is in front of or within a road works section. Looking only at a screenshot, one cannot infer how a bar is animated. For example, it is not possible to decide, whether the bar depicted in Figure 5 (left) is decreasing upwards or increasing downwards. This is one reason why it is important to use dynamically presented bars in an actual driving task to enable a comparison between the different bar types.

In Figure 6 we present an overview of the two experimental factors composition type and moving direction leading to four experimental conditions. Three snapshots illustrate the animation of each moving bar (from the leftmost to the rightmost). For each experimental condition a combination of an approach bar (either increase or decrease; either upwards or downwards) for approaching the road works was successively combined with its consistent progress bar (increase or decrease; all moving from left to right) within the road works. This resulted in four main experimental conditions (increasing upwards: inc_up; decreasing upwards; dec_up, increasing downwards: inc_dw; decreasing downwards: $d e c \_d w$; cf. Figure 6). 


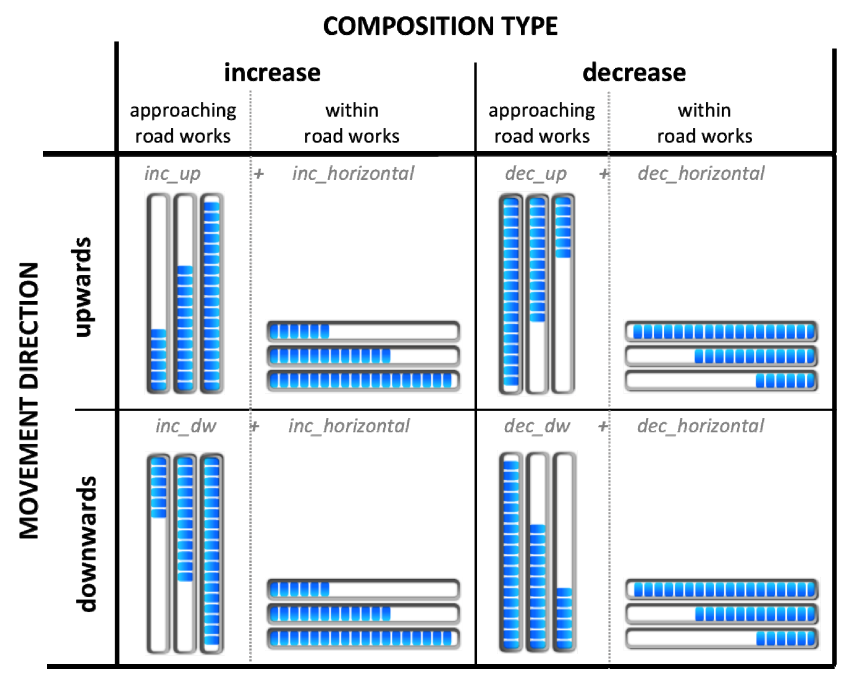

Fig. 6. Three snapshots illustrate the animation of each bar variant. Overview of four main experimental conditions: For each track an approach bar (either increase or decrease; either upwards or downwards) was combined with its consistent progress bar (increase or decrease; all moving from left to right).

As an informal pretest among eight colleagues had revealed, three bar types were favored or supported, whereas one variant (downward increase: inc_dw) was not appreciated by anybody. Furthermore, horizontal progress bars moving leftwards were evaluated as highly unsuitable. For reason of design completeness, we nevertheless kept the $i n c \_d w$ condition, but we omitted the leftwards progress bars - also analogous to desktop solutions.

As participants might behave differently when for the very first time encountering a bar in the road works scenario, we considered that counterbalancing presentation order of conditions would not to be sufficient with respect to our research questions. Therefore, we decided to use the rather unpopular variant $i n c \_d w$ as introductory condition. A second version of the inc_dw condition lacking the boundary line was generated and added as a fifth condition. The gray framing line is used for all four main bar variant conditions and indicates absolute minimum and maximum of bar states. Therefore, it might be a helpful reference for interpretation - especially for inexperienced users who do not know the bar range yet. To test this assumption, condition $i n c \_d w$ and $i n c \_d w \_f l(\mathrm{fl}=$ frameless) were alternately used between participants for either the introductory track (1) or the last track (5). Accordingly, the $i n c \_d w$ condition was experienced only half by novice participants and half by experienced participants. This kept the inc_dw condition comparable with the remaining three major experimental conditions (inc_up, dec_up, dec_dw), which were only assigned to middle tracks (2-4). Order of five equivalent physical driving tracks (with slightly differing distances, see also Driving Task and Tracks section) remained constant. The assignment of the three middle experimental conditions to the fixed tracks (2-4) was counterbalanced between participants to control for learning or sequence effects as well. 
For each bar type (except the inc_dw_fl condition), an animated color-coded version of the approach bars was additionally presented to participants (see Figure 7). We investigated, whether participants would prefer color-coding representing proximity and accordingly urgency over plain-colored bars.

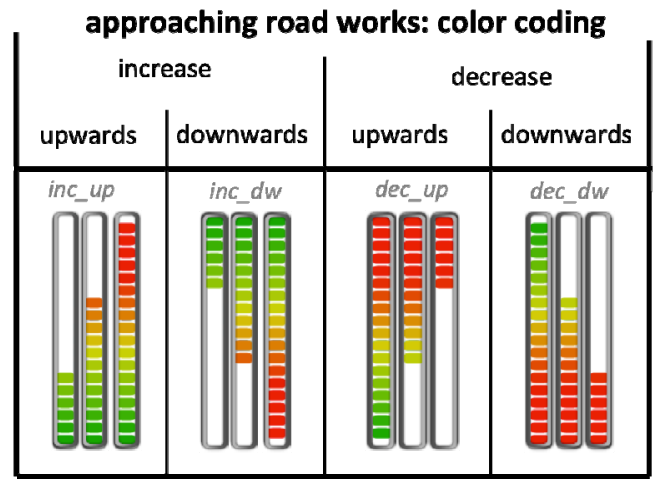

Fig. 7. Additional color-coded versions of approach bars presented to participants

\subsection{Design}

Two factors, movement direction (upwards, downwards) and composition type (increasing, decreasing) were manipulated within participants. A reference frame line as visual reference for minimum and maximum bar states was used for all four conditions. The inc_dw condition was additionally presented in a frameless version $\left(i n c \_d w \_f l\right)$. Half of the participants started with a $i n c \_d w$ track and finished with a $i n c \_d w \_f l$ track, whereas for the remaining half of the participants the order was reversed.

\subsection{Driving Task and Tracks}

Participants were instructed to drive as they would in a real world scenario. They should conform to German traffic rules (speed limits, taking the right lane whenever possible, not exceeding the current lane). Besides, they should finish each track as fast as possible. Moreover, they were informed that the small screen would provide helpful information about the upcoming traffic situations. Participants had to perform five equivalent two-lane motorway tracks without any other vehicles present. An example containing concrete distances between the events is depicted in Figure 8. All experimental tracks were designed according to the same scheme, but each track was created with different distances. This should prevent learning effects about the exact event positions and times. 


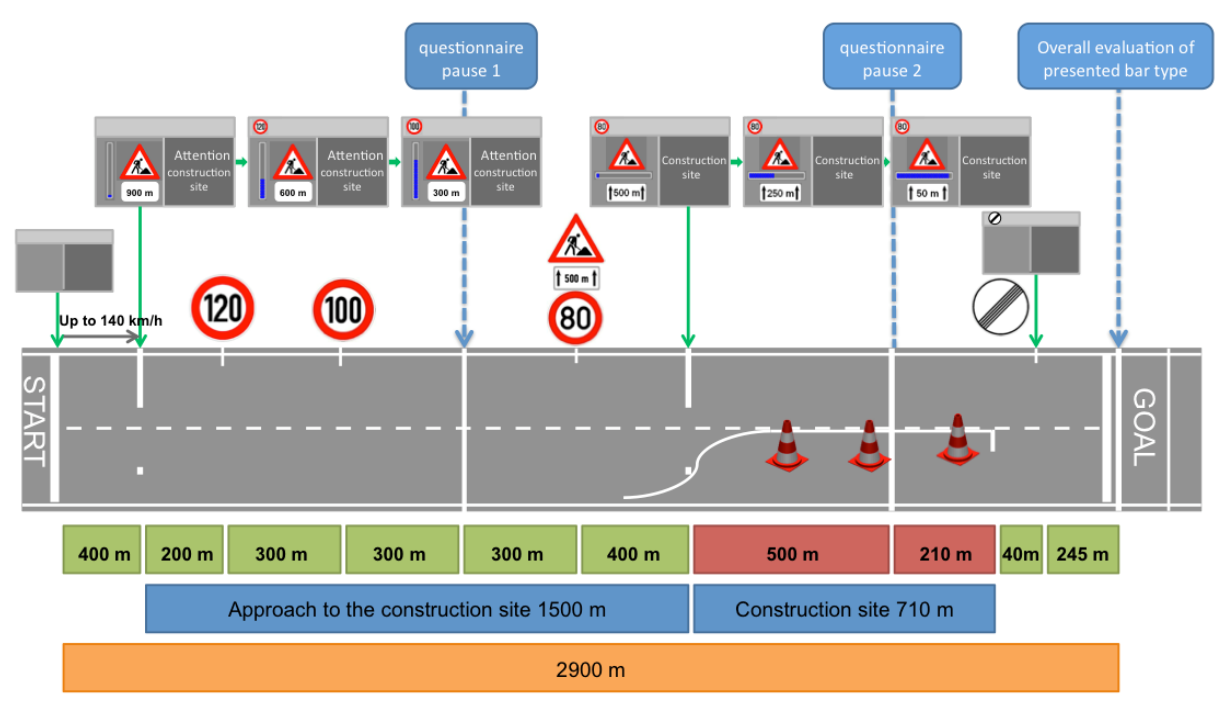

Fig. 8. Schematic overview of an experimental track containing speed limits, road works, and two pauses for questionnaires

After an acceleration phase without speed limit (acceleration to $140 \mathrm{~km} / \mathrm{h}$ ), a limit of 120 kilometers per hour requiring an immediate brake reaction was suddenly presented both on a road sign and on the small information screen. This speed limit was presented due to approaching road works. After a while, further speed limits showing 100 and 80 kilometers per hour were presented successively. When arriving at the road works it became obvious that the right lane was barred and participants had to change to the left lane. After passing the road works section at a speed of 80 kilometers per hour, the speed limit is revoked and participants should accelerate again. Shortly after, the track was finished. During each track, drivers were given notice of the road works on the small screen: Before arriving at the road works (from the first speed limit onwards), the remaining distance was shown by a numerical sign in combination with a vertical approach bar. Once the single lane within the construction work was entered, numbers combined with a horizontal progress bar displayed the remaining distance until the end of the road works segment. Having described participants, apparatus, experimental conditions, and the driving task, the next section will provide a description of the overall experimental procedure.

\subsection{Procedure}

After having been welcomed, participants filled out a first demographic questionnaire. The experimenter explained the driving task to the participant, and had her get familiar with the setup in a first practice drive on a straight road in the simulator. Subsequently, the participant completed a first baseline drive containing merely diverse speed limits but no road works or obstacles. Afterwards, the main experimental part was started, without the participant knowing anything about the upcoming road works 
in the first drive. The expectancy level certainly changed for the subsequent tracks, as both road works and speed limits reoccurred in each case. Nevertheless, exact locations were varied for each track, in order to prevent time-related expectancies and ignoring of the presented distance information. Within two breaks during each track and at the end of each track, participants had to fill out a questionnaire evaluating the current bar variant. Between plain-colored bars, color-coded bar variants were presented and evaluated as well. Following all experimental tracks, a second baseline drive had to be completed. After a final questionnaire participants were paid and said goodbye.

\subsection{Dependent Variables}

Most importantly, we assessed user preferences regarding bar presentation in general and intuitiveness of the presented bar alternatives. We were moreover interested, whether users interpret the meaning of some bars more consistently than the meaning of others. We assessed this by simply asking participants where they located themselves and the road works on the respective bar type during a short (driving) pause. Furthermore, we evaluated participants' gaze behavior onto the information screen, as this might vary for the different bar types. Low gaze distraction is on the one hand itself favorable for the driving context, and on the other hand it might serve as an implicit measure of presentation intuitiveness. Even though our driving task was rather simple, we recorded driving performance to control for any unforeseen disturbances or effects. For all dependent variables we intended to test whether for each factor main effects and interactions could be found, finally leading to a recommendation, which bar types seem(s) most suitable.

\section{$5 \quad$ Results}

As pointed out earlier, we wanted to generate a dynamic situation in a driving context that allows above all a valid subjective evaluation of different graphical distance estimation support variants. Possible differences in driving performance or glance behavior should be investigated as further dependent variables. Accordingly, we start with a rather detailed analysis of user preferences measured at different times during the experiment with the help of tailored questionnaires.

\subsection{User Preferences and Perceptions}

For a start, we present several results from the concluding questionnaire at the very end of the experiment. We asked participants their general opinion about the usage of progress bars. $75 \%$ of the participants appreciate progress bars as very useful or rather useful addition to numerical distance indicators, whereas only $25 \%$ of the participants rated them average, little or not useful at all. Hence, for the majority of drivers using bar indicators is valuable. Equivalent answers were given for using a differing alignment (vertical vs. horizontal) for approach and progress information. This leads to the assumption that different alignments predefined for different types of spatial 
information were assessed as a valuable premise. We state that this supports differentiation between approach and progress at a glance. In terms of use-cases, participants prefer progress bars for traffic events like traffic jams (mean $=4.1$ ), or obstacles (mean $=4.1$ ), whereas these bars are less appreciated for events like speed limits (mean $=2.8)$. An alternative design draft containing small symbols indicating car and hazard position received average ratings (mean $=3.3$ ), whereas modifying the small segments into arrows indicating the movement direction of the bar (analogous to the Falk screenshot in Figure 3d) received comparably positive ratings (mean = 3.7).

As a second step, we elaborate on the results for the vertical approach bar alternatives. During a short pause within each approach phase (see Figure 8), participants should mark on a printed screenshot of the respective bar where they located themselves, as well as the beginning of the road works. For upwards-moving bars we initially considered the road works to be represented by the upper frame line. For the downwards-moving bars, we initially considered road works to be represented at the bottom frame line. For all conditions, the driver's position was presumably represented within the frame line at the changeover from bar content to empty. Only if a participant indicated both positions accordingly her answer was coded as "correct". Participants could choose not to answer if they were unsure, but this was not counted as correct either. As becomes obvious in Figure 9, participants were quite sure about their answers for the upwards-moving bars leading to a high percentage of correct answers. Regardless of our initial hypothesis of "correct" positions, results reveal that participants immediately access concordant mental models for this type of visualization. On the contrary, major dissent occurred for the downwards-moving bars. We found a high proportion of at least partially incorrect answers and non-answers. This reveals an inconsistent interpretation (between subjects) and uncertainty for bars moving downwards. A Chi-Square test confirmed the improved performance levels for two upward compared with two downward approach bars, $\chi^{2}(1)=60.33, p<.05$.

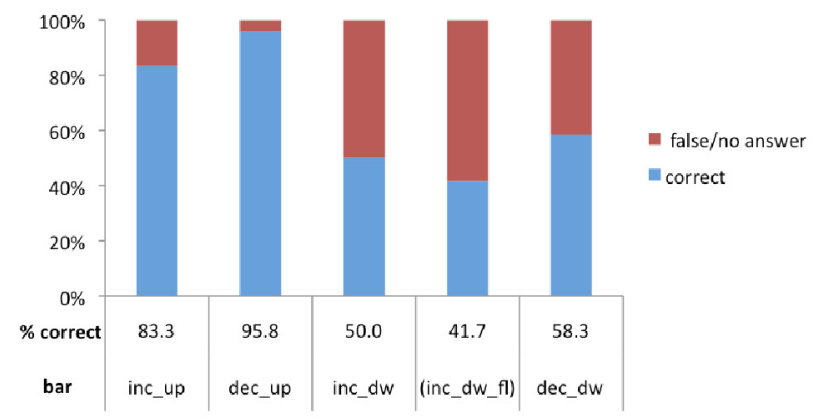

Fig. 9. Correct (red) versus at least partly incorrect (false or no answer; blue) answer percentage to the question: "Where are your car and the beginning of the road works located on this bar?". Results indicate consistency of bar assessment with xy.

Furthermore, participants were asked both during the test tracks as well as at the very end of the experiment, how intuitive they rated each graphical distance 
presentation from 1 - not intuitive to 5 - very intuitive. Figure 10 shows the results for the later ratings using a stacked bar graph ${ }^{2}$.

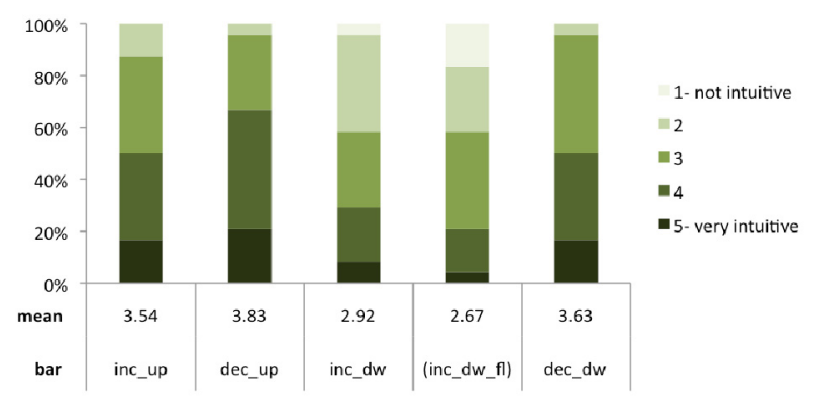

Fig. 10. Stacked bar graph illustrating the percentage of participants rating each approach bar from 1 - not intuitive to 5 - very intuitive. Darker bars indicate higher intuitiveness ratings.

A Repeated Measures MANOVA (i.e., we used the multivariate approach to the repeated measures analysis; see, e.g. [11, 12] with the factors movement direction (upwards vs. downwards) and composition type (increase vs. decrease) was conducted for the four main bar types. Movement direction had a significant influence on ratings with upwards moving bars being rated higher than the downward ones, $F(1,23)=12.49, p<.01$. However, neither composition type, $F(1,23)=2.34, p=.14$ nor the interaction revealed significant effects, $F(1,23)<1$, ns. Another comparison targets the factor reference frame line, which has been altered between bar inc_dw and $i n c \_d w \_f l$. Even though the order (first vs. last track) was counterbalanced, ratings did not show any significant difference $t(23)=-.77, n s$. However, relatively low ratings for a bar increasing downwards (inc_dw) might have concealed further negative influence of a missing reference frame (floor effect). Anyway, we would suggest using a frame line, as it does not worsen results. According to the results above, upwardsmoving bars (inc_up, dec_up) score higher than downwards-moving bars. For the two upwards bars, we furthermore wanted to investigate, whether additional color-coding (see Figure 7) would influence subjective assessment. Accordingly, we conducted a Repeated Measures MANOVA on bar ratings, with the factors composition type (inc_up vs. dec_up) and color-coding (with vs. without). A significant main effect of color-coding, $F(1,23)=9.31, p<.01$, indicates that participants clearly prefer color coding. Neither the other main effect of composition, $F(1,23)=2.46, p=.13$, nor the interaction, $F<1$, ns., led to significant results.

Beyond the four vertical approach bars, we present results for the corresponding two bars for progress within the road works (decreasing horizontal or increasing horizontal). During another short pause within the road works section (see Figure 8), participants should mark on a printed screenshot of the respective bar where they

\footnotetext{
${ }^{2}$ The later overall ratings did not differ compared with the earlier ratings, $F(1,20)=2.64$, ns. The interaction between point of time and bar variants also missed the level of significance, $F(4,17)=2.42, p=.09$. Accordingly, the pattern of ratings does not change significantly over time and it is sufficient to only present the later ratings in detail.
} 
perceived themselves, as well as beginning and end of the road works. For both bar types we considered the beginning of the road works to be represented by the very left frame line and the end at the very right. Consistent with the approach bars, the driver was presumably represented within the frame line at the changeover from bar content to empty. If participants were unsure about their assessment, they could also give no answer. Results were coded as "correct" if all position indications were as we had expected. The percentage of correct answers about the car's and the road works' position was relatively high for the decreasing (83\%) as well as for the increasing horizontal bar variant $(88 \%)$. For the frameless variant (horizontally increasing), rather inconsistent interpretations were achieved (63\%). To conclude, both framed progress bars were interpreted quite consistent with our initial assumption. These findings do at least not contradict our initial assumption that preselecting progress bars moving rightwards is feasible.

When having a look at the subjective ratings about the intuitiveness of progress bar types (cf. Figure 11), a significant tendency in favor of the decreasing progress bar (dec_ri), compared with the increasing progress bar (inc_ri) can be identified, $t=2.15, p<.05$. A comparison of both increasing bar types revealed significantly better ratings for the version with frame line (inc_ri) compared with the frameless bar $($ inc_ri_fl), $t(21)=2.57, p<.05$.

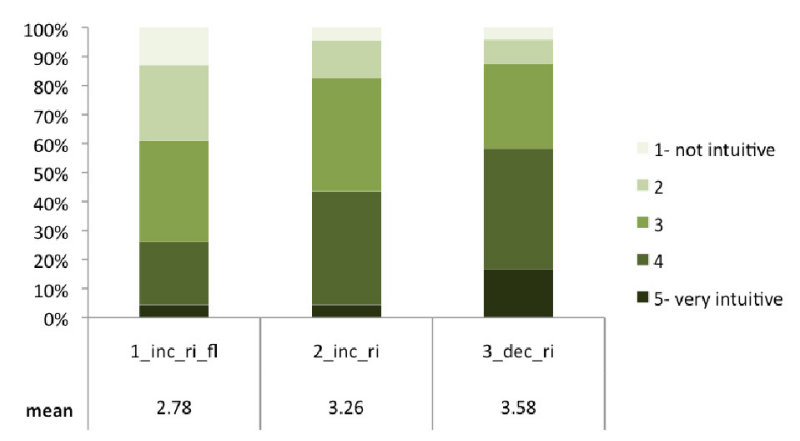

Fig. 11. Stacked bar graph illustrating the percentage of participants rating each progress bar from 1 - not intuitive to 5 - very intuitive. Darker bars indicate higher intuitiveness ratings.

\subsection{Gaze Behavior}

Besides gathering subjective ratings, also participants' gaze behavior onto the small information screen was recorded and analyzed, as glance behavior can serve as a valuable indicator for visual distraction from the road. We recorded average glance duration, maximum glance duration, number of glances during each track, and the proportion of time that participants glanced onto the small screen while driving. Note that results only labeled according to the five vertical bar variants (see Table 2) but they also include the horizontal bar increasing or decreasing consistently in each track. 
Table 2. Average fixation duration, number of glances, maximum glance duration, and percentage of time looking at the information screen for the five tracks

\begin{tabular}{|l|c|c|c|c|c|c|c|}
\hline Condition & $\boldsymbol{i n c \_ u p}$ & $\boldsymbol{d e c \_ u p}$ & $\boldsymbol{i n c \_ d \boldsymbol { w }}$ & $\boldsymbol{i n c _ { - } d w \_ f l}$ & $\boldsymbol{d e c} \_\boldsymbol{d} \boldsymbol{w}$ & BL 1 & BL 2 \\
\hline $\begin{array}{l}\text { Average glance } \\
\text { duration [ms] }\end{array}$ & 552 & 533 & 507 & 568 & 510 & 420 & 346 \\
\hline $\begin{array}{l}\text { Number of } \\
\text { glances }\end{array}$ & $\mathbf{2 2 . 4}$ & $\mathbf{1 9 . 8}$ & $\mathbf{2 1 . 0}$ & 23.5 & $\mathbf{1 8 . 6}$ & 8 & 7 \\
\hline $\begin{array}{l}\text { Maximum glance } \\
\text { duration [ms] }\end{array}$ & 1282 & 1236 & 1289 & 1286 & 1209 & 792 & 567 \\
\hline $\begin{array}{l}\text { Gaze \% of } \\
\text { driving time }\end{array}$ & $\mathbf{1 0 . 4}$ & $\mathbf{8 . 7}$ & $\mathbf{9 . 3}$ & 10.5 & $\mathbf{8 . 5}$ & 3.9 & 3.5 \\
\hline
\end{tabular}

First of all, we compared the initial and final baseline drives with an average of all four experimental conditions for all dependent variables. In the baseline drives merely the current speed limits were presented on the small screen according to the road signs in the simulator. Therefore, we expected lower values of gaze measurement for baseline tracks. This was confirmed for all collected parameters, $F(1,13)>12.79, p<$ .001 , for 14 out of 24 participants. Consistent with the implication of this finding, the remaining 10 participants did not glance onto the small screen at all in at least one of the baseline conditions, and therefore had to be excluded from the comparison above.

Most important we wanted to have a closer look at the two experimental factors movement direction and composition type. Analogous to the self-reported metrics in user preferences section, we entered all gaze metrics (average glance duration, maximum glance duration, number of glances, gaze percentage) of the four main bar alternatives into a two-way Repeated Measures MANOVA. We did not receive any significant effects for the average glance duration, $F(1,23)<1.57$, n.s., and for the maximum glance duration, $F(1,23)<1$, n.s. This indicates that the duration of single glances does not vary between our variants. However, most interestingly the number of glances per track, $F(1,23)=14.05, \mathrm{p}<.001$, as well as the overall percentage of experimental time spent with looking at the small information screen, $F(1,23)=7.25, p<.05$, showed a consistent main effect of composition type: Decreasing bars lead to less glances and less overall glancing time on the small information screen (cf. Figure 12). Neither significant main effects for movement direction, nor significant interactions were found. To sum up, participants rated the bars differently rather with respect to movement direction, but for gaze behavior the composition type seems to be more decisive.
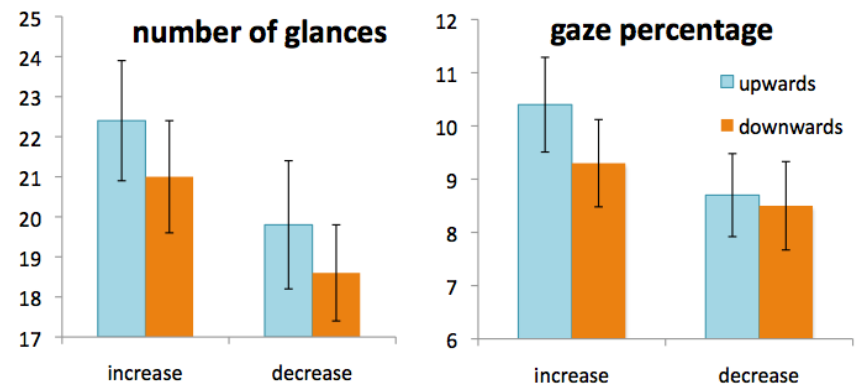

Fig. 12. Number of glances and percentage of gazes onto the small information screen during a track. Decreasing bars lead to significantly lower visual distraction than increasing bars. 


\subsection{Driving Performance}

Even though the driving task was very simple and all the tracks were designed to be similar, we investigated driving performance to control whether any unforeseen disturbance or confound had influenced driving behavior. Reaction times upon speed limits, deviation from the current speed limit, and deviation from a predefined ideal line (drive in the middle of the respective lane, fast lane change when necessary) were logged. Thereby, Neither reaction times upon speed limits, nor deviation of the ideal line, nor deviation of the current speed maximum revealed any significant differences, all $F^{\prime} s(1,23)<1, n s$. In summary, as expectable in this scenario no performance differences could be observed for the presented bar alternatives in the driving simulation scenario.

\section{Discussion, Conclusion, and Future Work}

Our results imply that participants appreciate the distinction of vertical approach bars and horizontal progress bars. For approach bars, upwards-moving bars are preferred over downwards-moving ones, whereas composition type ratings do not differ significantly. However, in gaze-based metrics decreasing bars induced less visual distraction from the primary driving task. This might occur because decreasing bar segments themselves contain all necessary spatial information without requiring a comparison with a further reference (e.g. frame line). Furthermore, for horizontal progress bars the decreasing bar compatibly received higher ratings as well. Moreover, color-coding leads to higher intuitiveness ratings, but obviously designers need to consider the trade-off between potential benefit and increased visual complexity in each individual case.

According to our findings, we suggest to use an approach bar decreasing in an upward direction. Spatial progress within a traffic event should consistently be presented via a horizontal bar decreasing to the right (see also Figure 13).

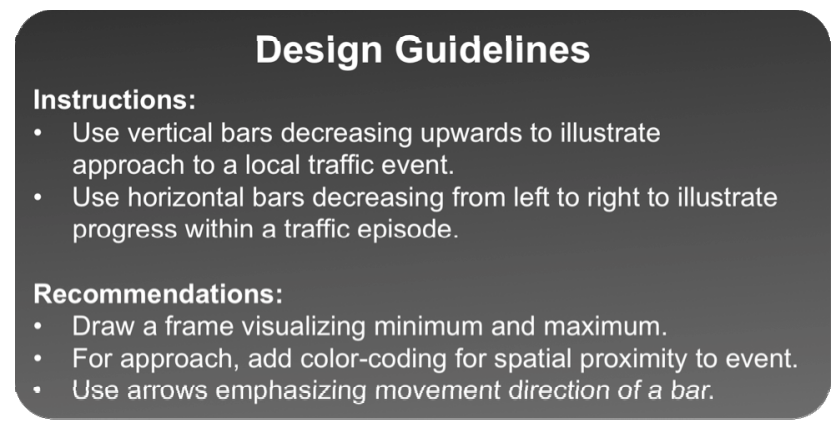

Fig. 13. Design guidelines for graphical bars representing spatial information when approaching local traffic events or for current progress within a traffic event section

On account of these guidelines, we designed graphical bars visualizing spatial approach and progress for the $\operatorname{sim}^{\mathrm{TD}}$ system that has been introduced earlier. Figure 14 
depicts example screenshots containing a final solution for approach and progress bars. In the field operational test the bars are consistently used for all appropriate usecases, e.g. obstacle warnings, traffic jam ahead warning, road signs.

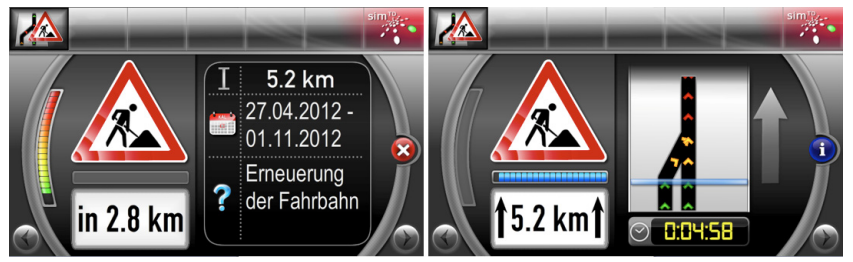

Fig. 14. Official $\operatorname{sim}^{\mathrm{TD}}$ screenshots of road works information containing a color-coded approach bar (left) and a single-colored horizontal progress bar (right)

Our results are a first step towards a consistent common solution for assisting the driver with graphical distance and progress information. Agreed design guidelines for human interface designers in the automotive domain would help users to immediately comprehend the presented spatial information in diverse in-vehicle information systems at a glance and with minimal cognitive effort. However, further research is needed to quantify benefits from the most suitable bar version compared with no graphical distance indicator. Even though we found out that the majority of drivers prefer bars in our study, we still lack knowledge, whether a well-designed bar should be prescribed for all appropriate systems. For this comparison of driver distraction we would recommend a more realistic driving environment providing more ecologic validity. Accordingly, differences in gaze behavior and driving performance would become more obvious - preparing the ground for investigation of additional moderators like gender or prior experience.

Acknowledgements. This work was funded within the project $\operatorname{sim}^{\mathrm{TD}}$ by the German Federal Ministries of Economics and Technology as well as Education and Research, and supported by the Federal Ministry of Transport, Building and Urban Development. EIT ITC Labs supported OpenDS via the activity Apps for Your Car (AIMS 13131) in the context of the project GetHomeSafe within the scope of the Seventh Framework Program of the European Commission.

\section{References}

1. "Driver Focus-Telematics Working Group": Statement of Principles, Criteria and Verification Procedures on Driver Interactions with Advanced In-Vehicle Information and Communication Systems (2006), http: / / www . autoalliance.org/

2. Hamada, K., Yoshida, K., Ohnishi, K., Koppen, M.: Color Effect on Subjective Perception of Progress Bar Speed. In: Third IEEE International Conference on Intelligent Networking and Collaborative Systems, pp. 863-866. Institute of Electrical and Electronics Engineers (IEEE), Fukuoka (2011) 
3. Harrison, C., Yeo, Z., Hudson, S.E.: Faster Progress Bars: Manipulating Perceived Duration with Visual Augmentations. In: Proceedings of the 28th Annual SIGCHI Conference on Human Factors in Computing Systems, pp. 1545-1548. ACM, New York (2010)

4. Harrison, C., Amento, B., Kuznetsov, S.: Rethinking the progress bar. In: Proceedings of the 20th Annual ACM Symposium on User interface Software and Technology, pp. 115-118. ACM, New York (2007)

5. Hsu, S., Le Prado, C., Natkin, S., Liard, C.: New Type of Auditory Progress Bar: Exploration, Design and Evaluation. In: Stephanidis, C. (ed.) UAHCI 2007 (Part II). LNCS, vol. 4555, pp. 868-877. Springer, Heidelberg (2007)

6. Brewster, S.A., King, A.: The design and evaluation of a vibrotactile progress bar. In: First Joint Eurohaptics Conference and Symposium on Haptic Interfaces for Virtual Environment and Teleoperator Systems, pp. 499-500. Institute of Electrical and Electronics Engineers (IEEE), Pisa (2005)

7. Myers, B.A.: The importance of percent-done progress indicators for computer-human interfaces. In: Borman, L., Curtis, B. (eds.) Proceedings of the SIGCHI Conference on Human Factors in Computing Systems, pp. 11-17. ACM, New York (1985)

8. Math, R., Mahr, A., Moniri, M.M., Müller, C.: OpenDS: A new open-source driving simulator for research. In: Adjunct Proceedings of the 4th International Conference on Automotive User Interfaces and Interactive Vehicular Appilcations, Portsmouth, NH, USA, pp. 7-8., http: / /www. auto-ui .org/12/

9. Paßmann, C., Schaaf, G., Naab, K.: simTD Project result: Selection of Functions, Deliverable D11.2 (2009), http: / / www . simtd. de

10. "the International Organization for Standardization": ISO/TR 16352: Road vehicles - Ergonomic aspects of in-vehicle presentation for transport information and control systems Warning systems (2005)

11. Dien, J., Santuzzi, A.M.: Application of repeated-measures ANOVA to high-density ERP datasets: A review and tutorial. In: Handy, T. (ed.) Event-Related Potentials: a Methods Handbook, pp. 57-82. MIT Press, Cambridge (2005)

12. O'Brien, R., Kaiser, M.: MANOVA method for analyzing repeated measures designs: An extensive primer. Psychological Bulletin 97, 316-333 (1985) 